\title{
Relationship between Computed Tompgraphy Pulmonary Angiography Findings and Clinical Findings Based on Wells Score in Acute Pulmonary Thromboembolism
}

\author{
Seyed Kamaledin Hadei $^{1}$ (i), Leila Molani ${ }^{2 * * i D}$, Zohreh Kahramfar ${ }^{3}$, Younes \\ Mohammadi $^{4}$ \\ ${ }^{I}$ Assistant Professor, Department of Radiology, School of Medicine, Hamadan University of Medical Sciences, Hamadan, Iran \\ 2 Resident, Department of Radiology, School of Medicine, Hamadan University of Medical Sciences, Hamadan, Iran \\ ${ }^{3}$ Assistant Professor, Department of Internal Medicine, School of Medicine, Hamadan University of Medical Sciences, \\ Hamadan, Iran \\ ${ }^{4}$ Associate Professor, Department of Epidemiology, School of Health, Hamadan University of Medical Sciences, Hamadan, \\ Iran \\ * Corresponding Author: Leila Molani, Department of Radiology, School of Medicine, Hamadan University of Medical \\ Sciences, Hamadan, Iran.Email: leilamolani@gmail.com
}

Received: 20.04 .2021

Accepted: 02.08.2021

How to Cite this Article:

Hadei SK, Molani L, Kahramfar Z, Mohammadi Y. Relationship between Computed Tompgraphy Pulmonary Angiography Findings and Clinical Findings Based on Wells Score in Acute Pulmonary Thromboembolism. Avicenna J Clin Med. 2021; 28(2): 71-78. DOI: 10.52547/ajcm.28.2.71

\begin{abstract}
Background and Objective: Acute pulmonary thromboembolism is an emergency disease that can lead to mortality if not diagnosed on time. Proper triage of patients based on clinical findings and the Wells Score will have a significant impact on the medical team's decision-making and patient management.

Materials and Methods: This descriptive cross-sectional study was conducted on 300 patients selected by the census sampling method. The patients were suspected of acute pulmonary thromboembolism referring to Farshchian Heart Hospital in Hamadan, Iran, within October 2017-March 2020. The cases considered a candidate for computed tomography (CT) pulmonary angiography were selected and compared regarding CT pulmonary angiography results and the risk of embolism based on Wells Score.

Results: According to the results of CT angiography, 91 (30.3\%) patients had acute pulmonary thromboembolism, $16.5 \%, 45.1 \%$, and $38.4 \%$ of which were in the low-risk, moderate-risk, and high-risk groups, respectively $(\mathrm{P}<0.001)$. Involvement of segmental and subsegmental branches, followed by the involvement of the pulmonary lobar branches, were the most common patterns of pulmonary artery involvement in acute pulmonary thromboembolism. Accompanying findings were significantly lower in patients with acute pulmonary thromboembolism than in patients with negative acute pulmonary thromboembolism $(\mathrm{P}<0.001)$.

Conclusion: There was a significant relationship between the result of CT pulmonary angiography and clinical findings in patients suspected of acute pulmonary thromboembolism. The careful examination of the clinical symptoms of patients and determination of the level of risk in such patients according to the Wells Score can lead to the more efficient management of the process of diagnosis and treatment of patients.
\end{abstract}

Keywords: Angiography, Acute Pulmonary Thromboembolism, Wells Score 


\title{
ارتباط بين يافتههاى سىتى آنزيوترافى يولمونرى و يافتههاى بالينى بر اساس معيار

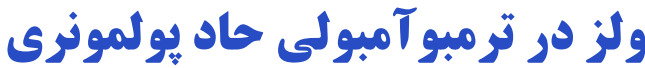

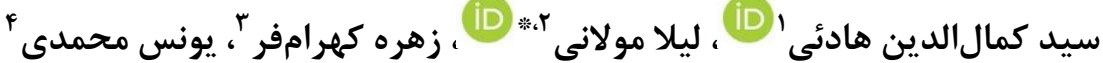

' ' استاديار، كروه راديولوزى، دانشكده يزشكى، دانشكاه علوم يزشكى همدان، همدان، ايران

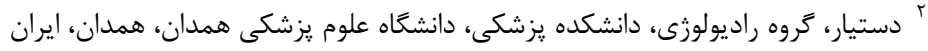

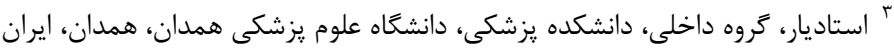

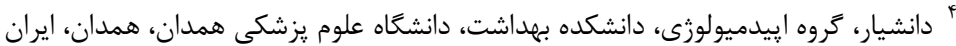
leilamolani@gmail.com : قويسنده مسئول: ليلا مولانى، كروه راديولورى، دانشكده يزشكى، دانشگاه علوم يزشكى همدان، همدان، ايران. ايميل

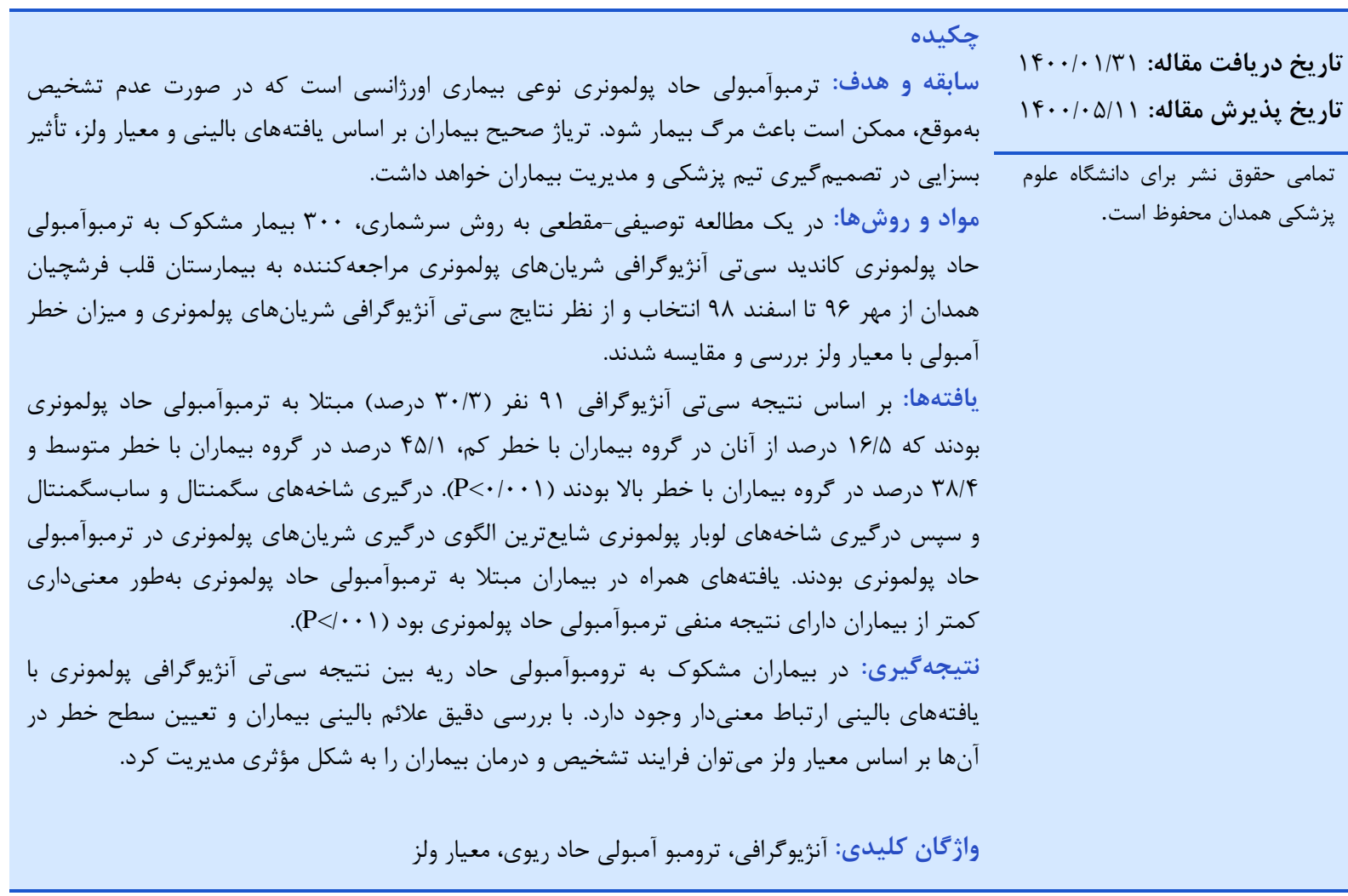

مقدمه

مهرم مطرح مىشود كه در صورت عدم تشخيص و درمان

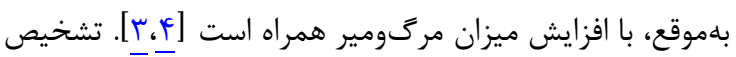

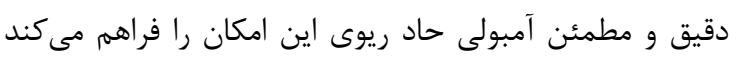

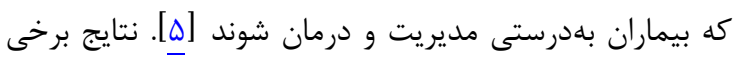

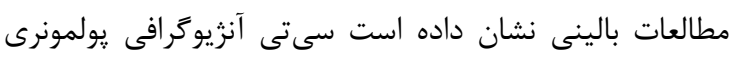
نوعى (CT Pulmonary Angiography: CTPA) تصويربردارى با كيفيت مناسب است كه بهعنوان انتخاب اصلى

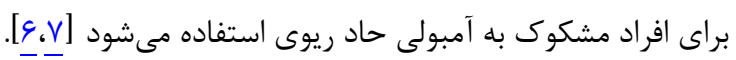

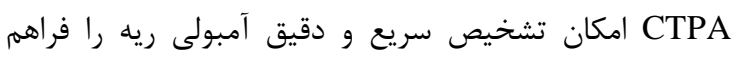

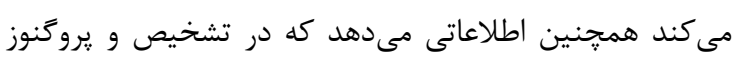
بيماران كمك مى كند. هر جند ميزان استفاده از
ترومبوآمبولى ريوى شرايط بالقوه كشندهاى است كه

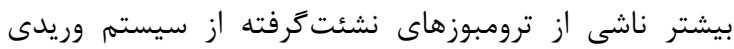

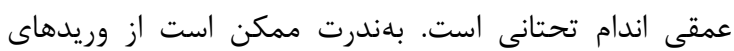

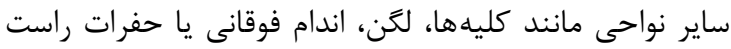

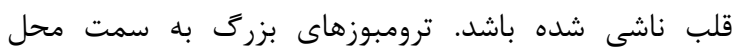

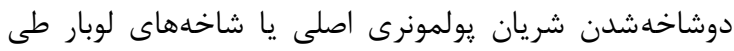

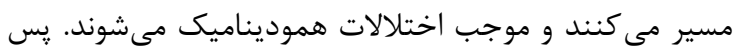

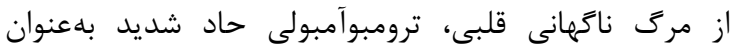

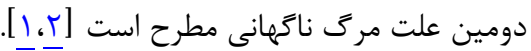

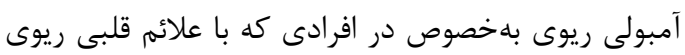

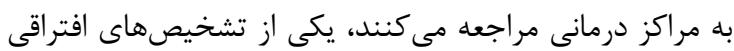


معيارهاى ولز براى تمامى بيماران محاسبه شد و بر اساس آن

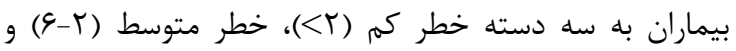

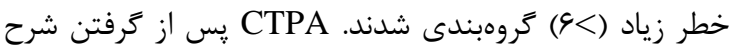
حال آسم فعال و حساسيت به ماده حاجب يا غذاهاى درون دريايى و

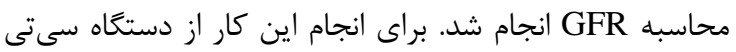

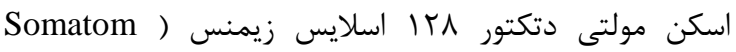
test استفاده شد. يس از انجام (Definition AS, Germany

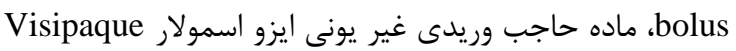

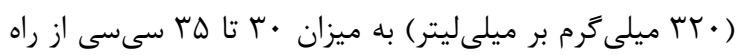

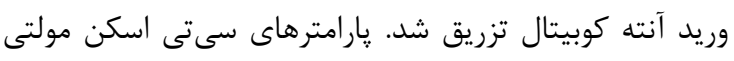
اسلايس به شرح ذيل بود:

$\mathrm{MA}=210, \mathrm{kv}=100$, slice thickness $=3 \mathrm{~mm}$, collimation: $128 \times 0.6 \mathrm{~mm}$, reconstraction increment $=0.6 \mathrm{~mm}$

بازسازى تصاوير در برشهاى ظريف ع| • تا ا ميلىمتر در

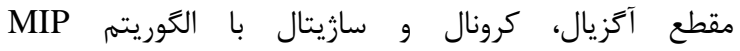
MPR و (Maximum Intensity Projection) (Multiplanar Reformation) ميمنس توسط راديولوزيست مجرب تفسير و ميزان دركيرى عروق يولمونر مشخص شد. ترومبوآمبولى حاد

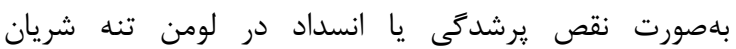

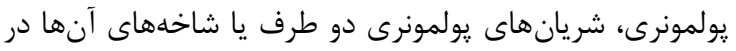
نظر گرفته شد (شكل () ). دادههاى بيماران يس از گردآورى، با نرمافزار

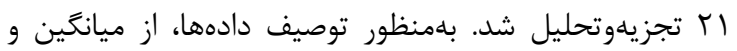

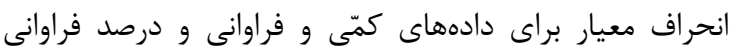

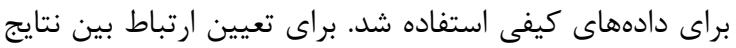

CTA

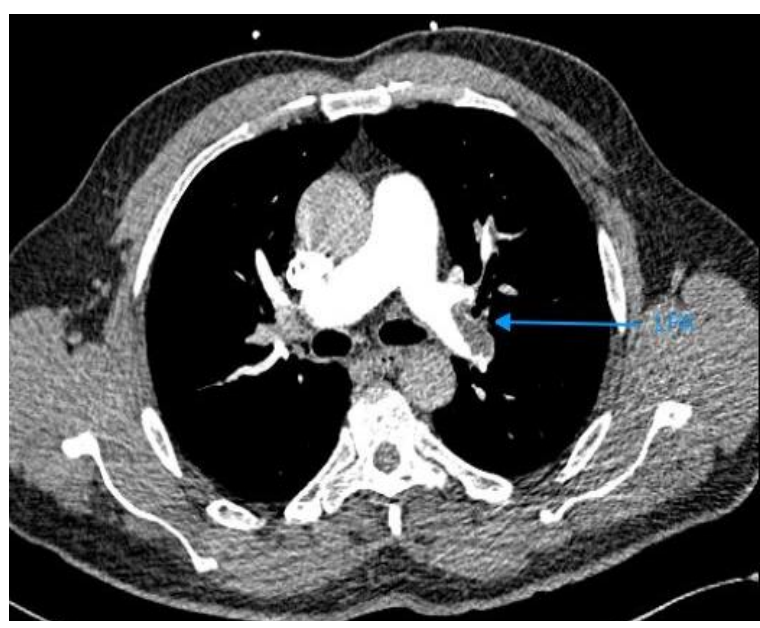

شكل ا: ترومبوآمبولى حاد ريوى در يك بيمار مرد اه ساله

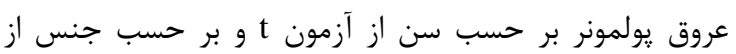

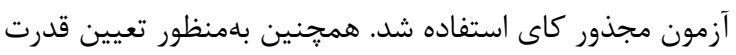

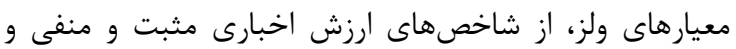

يافته است، همجنان كمتر از • ا درصد از موارد آمبولى ريه

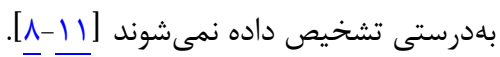

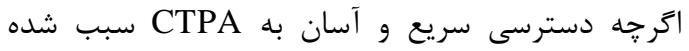

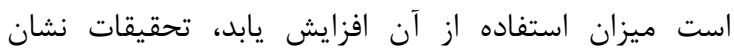

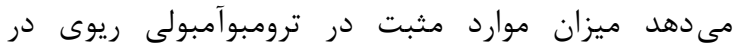

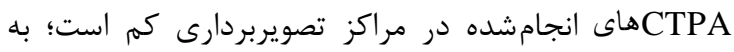

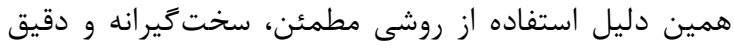

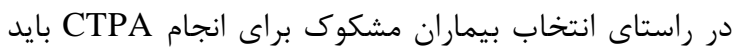
در دستور كار يزشكان قرار گيرد. معيار ولز (Wells Score)

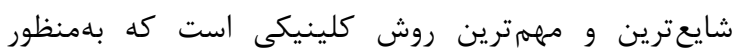

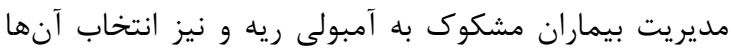

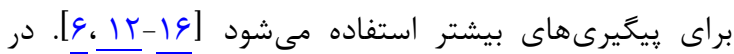
سال 1991 ولز قواعد ويزهاى را بر اساس علائم بالينى و نيز

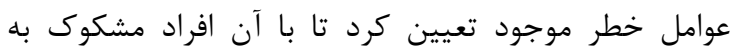
آمبولى ريه بهطور دقيقترى شناسايى شوند [l IV]

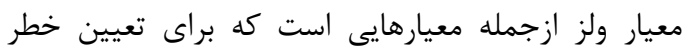

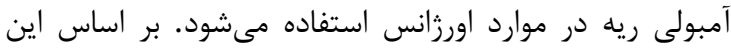

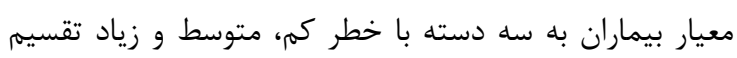

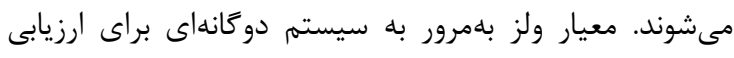

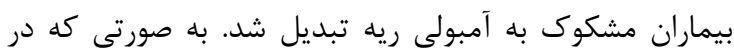

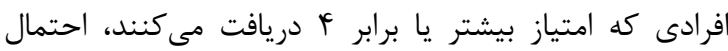

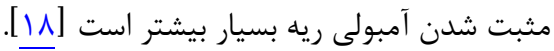

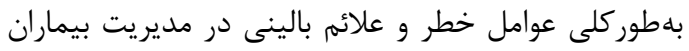

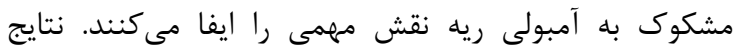
تحقيقات نشان مى دهد در بيمارانى كه هيجيك از معيارهاى ولز

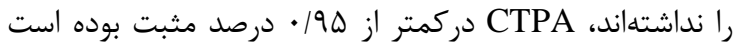

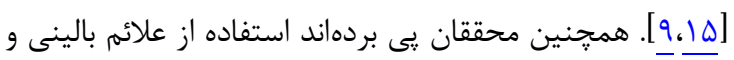

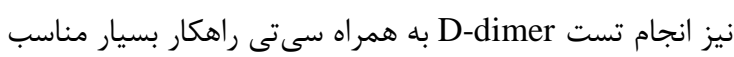

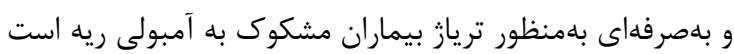

اين مطالعه با هدف تعيين ارتباط نتايج سىتى آنزيوگرافى

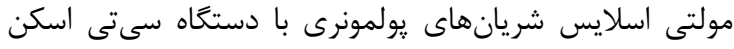

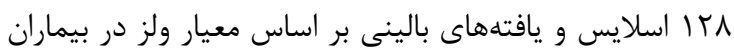

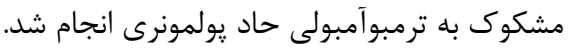

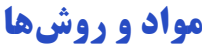

در اين مطالعه توصيفى-مقطعى، به روش سرشمارى تمام

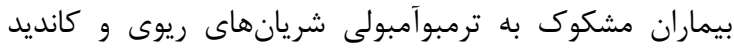
CTPA

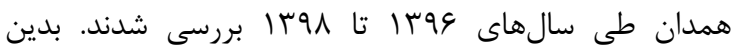
منظور شكايت اصلى بيمار، سابقه DVT و آمبولى رئى ريه، بيمارى

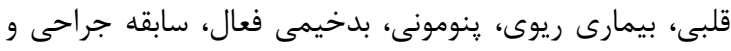

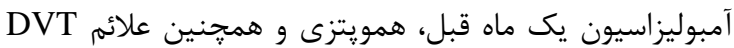

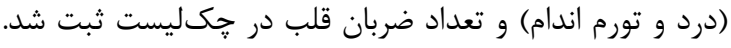




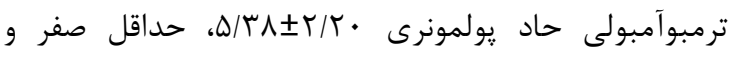
نسبت درستنمايى مثبت و منفى استفاده شد.

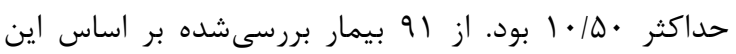

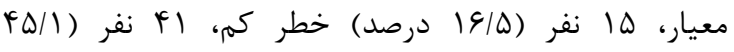
يافته ها

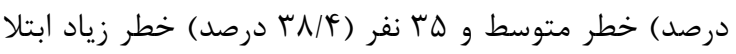

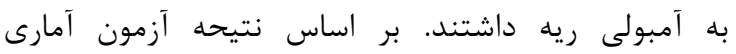

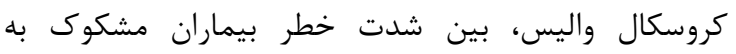

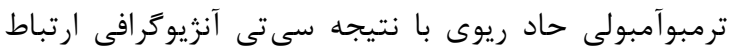

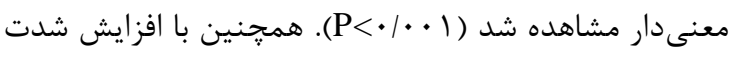

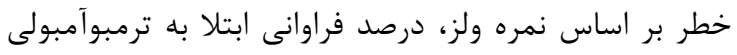

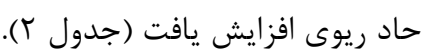

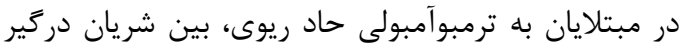

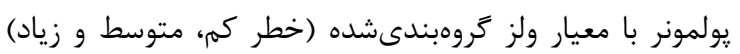

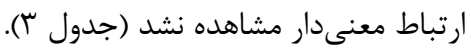

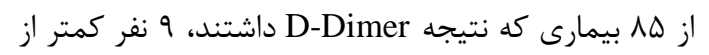

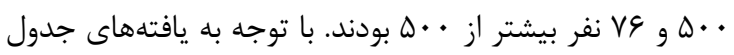

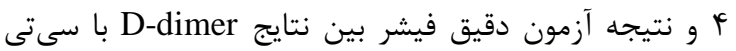
آنزيوكرافى اختلاف معنى دار مشاهده نشد.

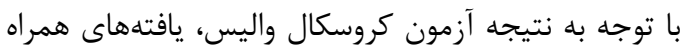

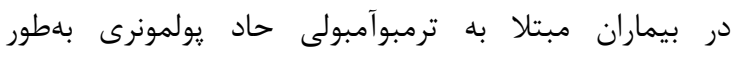

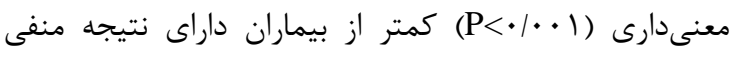

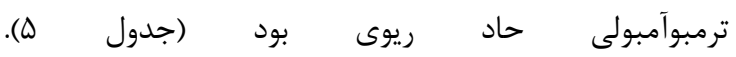

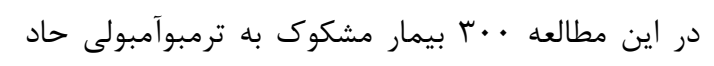

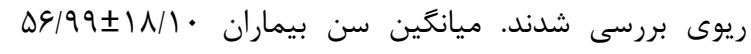

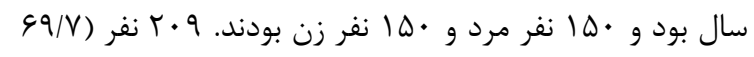

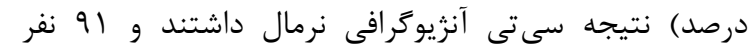

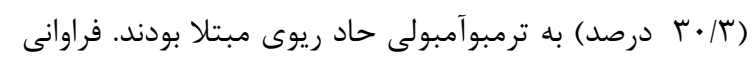

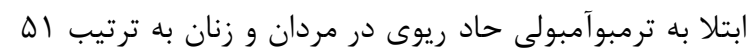

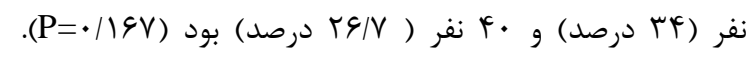

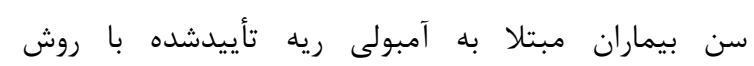

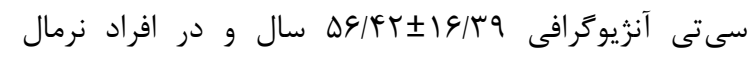

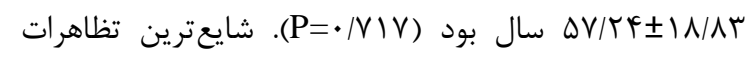

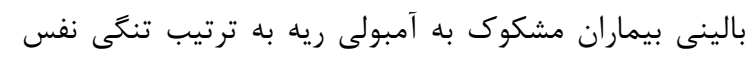

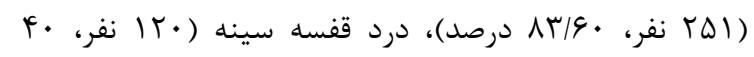

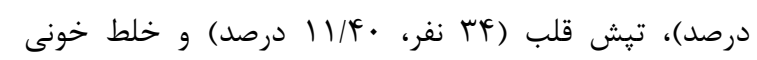

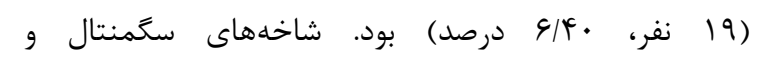

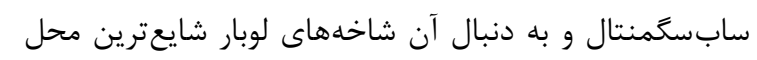

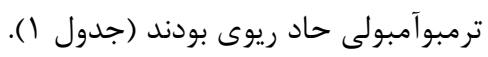

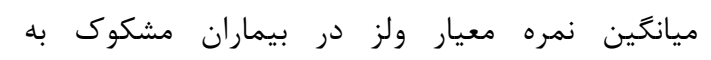

\begin{tabular}{|c|c|c|}
\hline درصد & 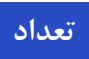 & شريان ريوى در تير \\
\hline$r \Delta / 1$ & (i) & شاخههاى سخمنتال و سابسخمنتال \\
\hline$r \Delta / r$ & r & شاخههاى لوبار و سَمنتال و سابسَمنتال \\
\hline$r \cdot / 9$ & 19 & شريان يولمونرى راست يا حِ و شاخههاى لوبار و سحَمنتال و سابسَمنتال \\
\hline$r / r$ & r & شريان يولمونرى راست يا حِ و شاخههاى لوبار \\
\hline$r / T$ & r & تنه اصلى شريان يولمونرى \\
\hline $1 / 1$ & 1 & شريان يولمونرى راست يا جٍ \\
\hline $1 / 1$ & 1 & شاخههاى لوبار \\
\hline $1 / 1$ & 1 & شريان يولمونرى راست يا حِ و شاخههاى سحَمنتال و سابسَمنتال \\
\hline $1 \cdots$ & 91 & مجموع \\
\hline
\end{tabular}

جدول r: توزيع فراوانى معيار ولز بر اساس سطح خطر در بيماران مشكوى به ترومبوآمبولى حاد ريوى (PTE)

\begin{tabular}{|c|c|c|c|c|}
\hline \multirow[b]{2}{*}{ ارزش P P } & \multicolumn{3}{|c|}{ نتيجه سى تى آنزيويَرافى } & \multirow[b]{2}{*}{ معيار ولز } \\
\hline & تعداد (درصد) & $\begin{array}{c}\text { PTE } \\
\text { تعداد (درصد) }\end{array}$ & 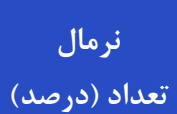 & \\
\hline \multirow{4}{*}{$<\cdot|\cdot \cdot|$} & $(1 \cdots) 99$ & $(\mid \Delta / \Gamma) \backslash \Delta$ & $(\Lambda F / \Lambda) \wedge F$ & خطر كم > \\
\hline & $(1 \cdot \cdot) \mid \Delta T$ & $(T V \mid \cdot)^{|r|}$ & $(V \Psi / \cdot)) 11$ & خطر متوسط Y-Y \\
\hline & $(1 \cdots)+q$ & $(V I / f) r^{\prime} \Delta$ & $(T N / Y) \mid Y^{4}$ & خطر زياد >9 \\
\hline & $(1 \cdots) \mu \cdot$ & $(r \cdot / r) 91$ & $(99 / V) r \cdot q$ & مجموع \\
\hline
\end{tabular}

جدول با: توزيع الكوى دركيرى شريانهاى يولمونرى در بيماران مبتلا به ترمبوآمبولى حاد ريوى بر حسب خطر ابتلا معيار ولز 


\begin{tabular}{|c|c|c|c|c|c|}
\hline P ارزش P & تعداد (درصد) & تعداد (درصد) زياد & تعداد (درصدر ابتلاد & تعداد (درصد) & شريان ريوى درَير \\
\hline \multirow{8}{*}{$.19 Y \wedge$} & $\left.(1 \cdots)\right|_{1}$ & $(r \varepsilon / \varepsilon) \backslash \Delta$ & $(\mathcal{F} / \Lambda) Y$. & $(\mid f / \varepsilon) \&$ & شاخههاى سخمنتال و سابسَمنتال \\
\hline & מ r (1) & $(\Delta T / T) \mid T$ & $(f+/ \Delta))$. & $(\boldsymbol{F} / \Psi))$ & شاخه لوبار و سگمنتال و سابسگمنتال \\
\hline & $(1 \cdot) 19$ & $(\Psi) / 9) \varphi$ & $(\uparrow \xi \mid \Lambda) \vee$ & $(\Psi) / \varepsilon) \varphi$ & 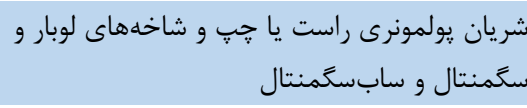 \\
\hline & $(1 \cdot \cdot)^{\mu}$ & $(T / \mu) 1$ & $(\varphi g / V) r$ & $(\cdot) \cdot$ & شريان يولمونرى راست يا حֶٍ و شاخههاى لوبار \\
\hline & $(1 \cdots)^{4}$ & $(\cdot) \cdot$ & $(\Delta \cdot / \cdot))$ & $(\omega \cdot / \cdot))$ & تنه اصلى شريان يولمونرى \\
\hline & $(1 \cdots) 1$ & $(\cdot) \cdot$ & $(\cdot) \cdot$ & $(1 \cdots) 1$ & شريان يولمونرى راست يا جٍٍ \\
\hline & $(1 \cdots) 1$ & $(1 \cdots))$ & $(\cdot) \cdot$ & $(\cdot) \cdot$ & شاخههاى لوبار \\
\hline & $(1 \cdots) 1$ & $(\cdot) \cdot$ & $(1 \cdots) 1$ & $(\cdot) \cdot$ & 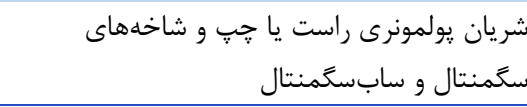 \\
\hline
\end{tabular}

\begin{tabular}{|c|c|c|c|c|}
\hline \multirow{2}{*}{ ارزش P } & \multirow{2}{*}{ مجموع تعداد (درصد) } & \multicolumn{2}{|c|}{ نتيجه سىتى آنزيو } & \multirow{2}{*}{ D-Dimer } \\
\hline & & PTE تعداد (درصد) & نرمال تعداد (درصد) & \\
\hline \multirow{3}{*}{$\cdot|r V|$} & $(1 \cdots) \vee \varepsilon$ & $(T / / \varepsilon) Y F$ & $\left(\varepsilon N / Y^{\mathcal{C}}\right) \Delta T$ & بيشتر از · . \\
\hline & $(1 \cdots)^{9}$ & $(11 / 1) 1$ & $(\Lambda \Lambda / 9) \wedge$ & كمتر از · •هـ \\
\hline & $(1 \cdots) \wedge \Delta$ & $(r q / F) r \Delta$ & $(\vee \cdot \mid \varepsilon) \varepsilon$. & مجموع \\
\hline
\end{tabular}

\begin{tabular}{|c|c|c|c|}
\hline ارزش P & فعى مثداد (درصد) & تعداد (درصد) تندال & يافته همراه \\
\hline \multirow{7}{*}{$<\cdot 1 \cdot \cdot 1$} & $(9 N / I) G 4$ & $(\mathcal{L} \cdot / V) \wedge \Delta$ & بدون يافته همراه \\
\hline & $(1 / 1) 1$ & $(V / T) \backslash Q$ & يلورال افيوزن \\
\hline & $\left(\mathcal{F} / \mathbb{F}^{\mathcal{F}}\right)^{\mathcal{F}}$ & $(1 \cdot) \times 1$ & كدورت ريه \\
\hline & $(T / T) Y$ & $(V / D) / T$ & بيمارى مزمن ريه \\
\hline & $(\mid Q / f) \mid f$ & $(Y / / D) F \Delta$ & يافتههاى CHF \\
\hline & r & $(T / F) D$ & يافتههاى PAH \\
\hline & $(\Delta / \Delta) \Delta$ & $(I T / F) Y G$ & بيش از يك يافته \\
\hline
\end{tabular}

زن باردار، T\&/ ب درصد از آنان بر اساس نتيجه سىتى

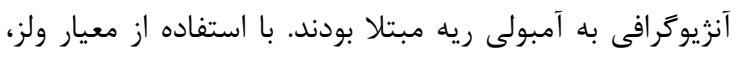

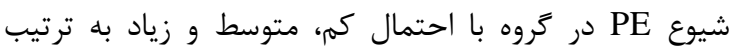

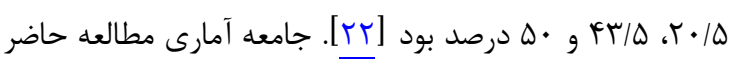

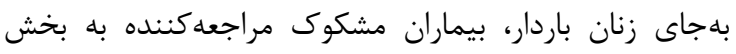

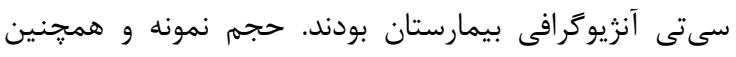

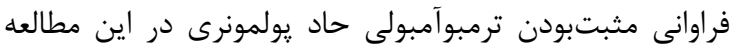

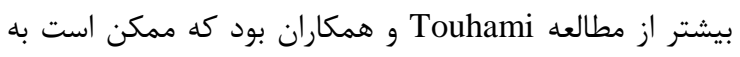

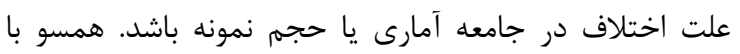
يافته هاى مطالعه Touhami و همكاران، در مطالعه حاضر نيز

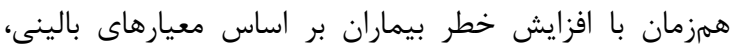

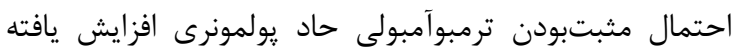

سىتى آنزيو گرافى ريه، اولين روش تصويربردارى براى

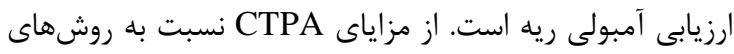

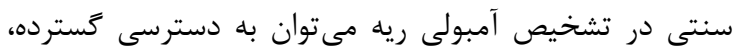

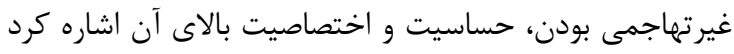

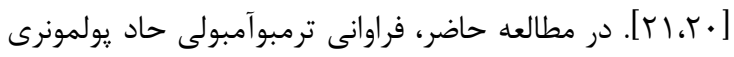

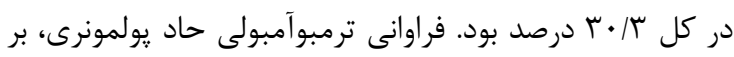

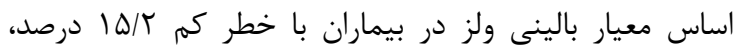

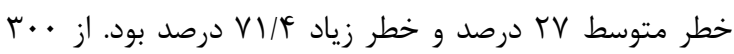

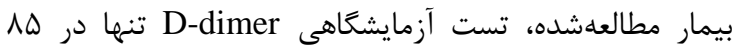

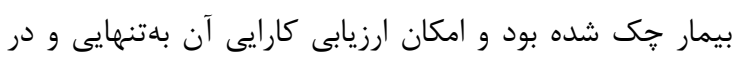
همراهى با معيار ولز مقدور نشد.

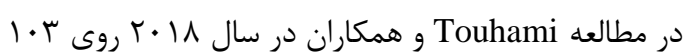


شيوه گروهبندى بيماران و ارزيابى خطر بر اساس معيارهاى دو

بالينى

مطالعه باشد.

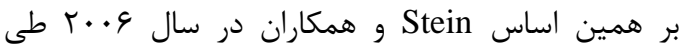

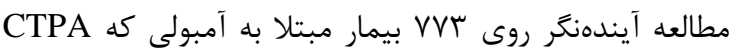

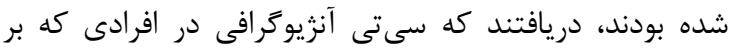

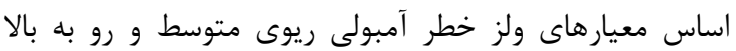

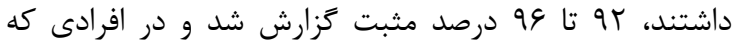

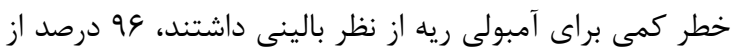

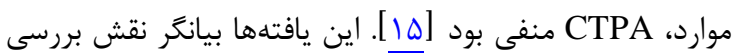

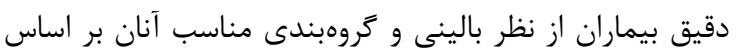

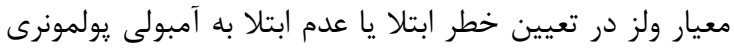

ارزش اخبارى مثبت CTPA در مواردى كه شك بالينى

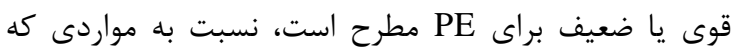

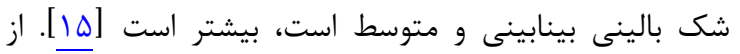

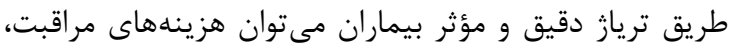

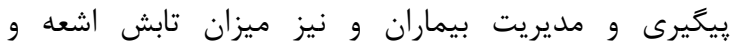

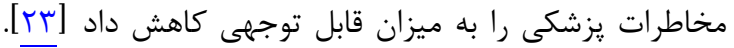

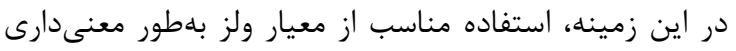

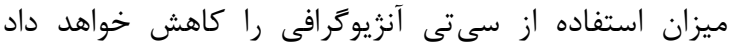

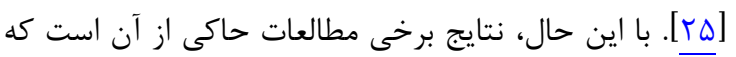

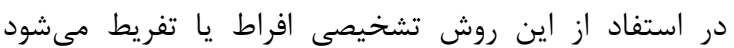

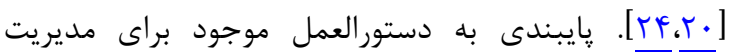

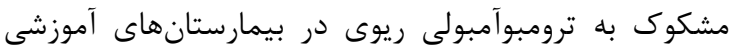

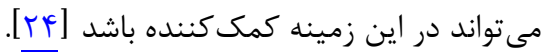

\section{نتيجه تيرى}

در بيماران مشكوك به ترومبوآمبولى حاد ريه بين نتيجه

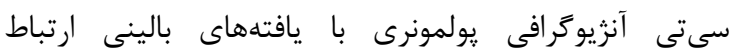

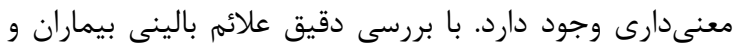

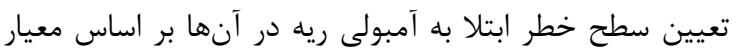
ولز مىتوان فرايند تشخيص و درمان بيماران را به شكل مؤثرى

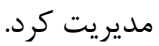

\section{تشكر و قلروانى}

اين مقاله بركرفته از پايان نامه دوره دستيارى تخصصى

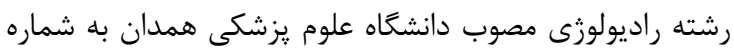

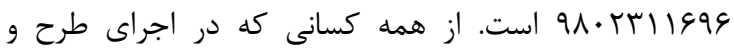
كردآورى دادهها مشاركت داشتند، تقدير و تشكر مىشود.

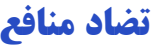
هيج گونه تعارضى از سوى نويسندگان كزارش نشده است.
Raji عملكرد تشخيصى CT آنزيوگرافى ريه با استفاده از نمره و

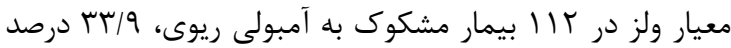

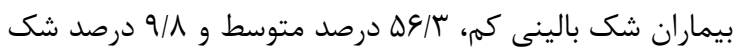

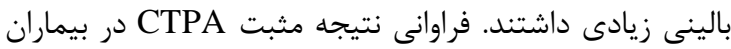

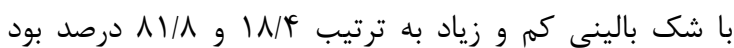

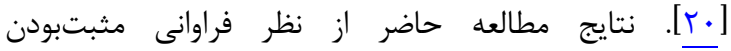

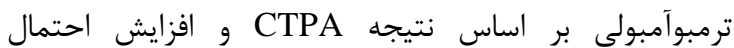
مثبتبودن ترمبوآمبولى حاد همزمان با افزايش خطر آن بر بر آن

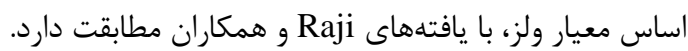

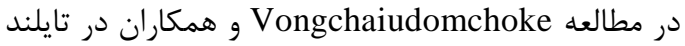

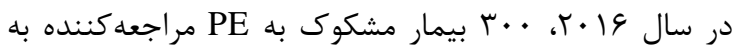

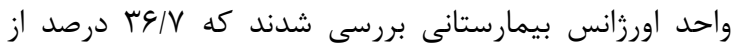

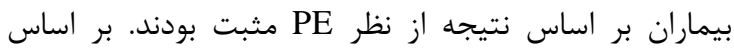

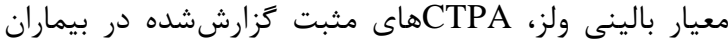

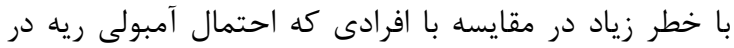

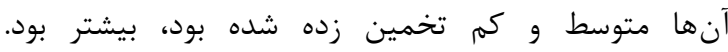

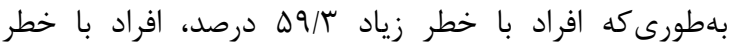

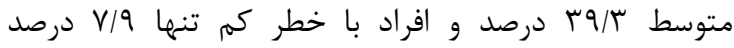
CTPA شيوه گروهبندى بيماران بر اساس معيار ولز مشابه مطالعه دماند Vongchaiudomchoke براى تشخيص ياراكلينيك از سىتى آنزيوكرافى استفاده شد.

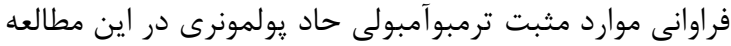

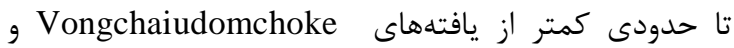

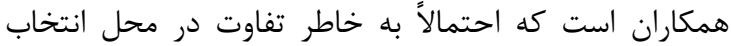
نمونهها است (بخش سىتى آنزيوگرافى در مقابل اورزانس)،

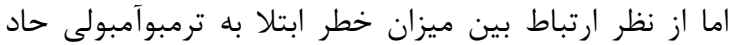

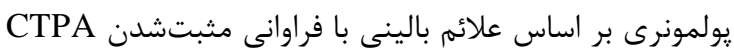
نتايج دو مطالعه همسو است. Molae تعيين استفاده مناسب از CTPA در بيماران مشكوك به دمران

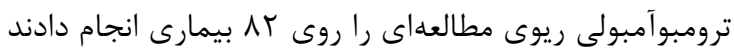

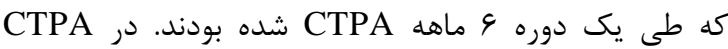
ميزان ترومبوآمبولى ريوى در بيماران با احتمال بالينى زياد

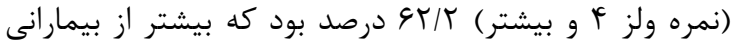

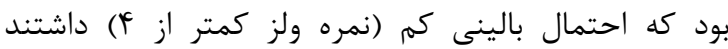

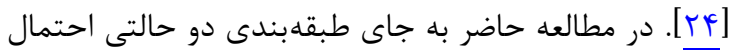
زياد و كم، از طبقهبندى سه حالتى به صورت خطر كم،

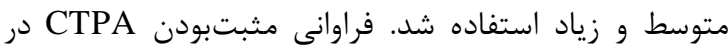
تحقيق حاضر بيشتر از مطالعه Molae و همكاران بود ( VI/F

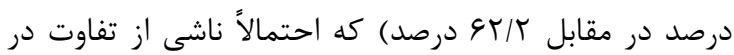




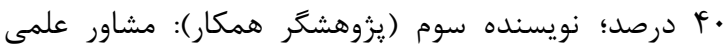

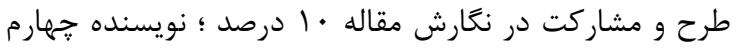

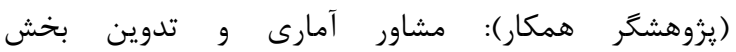

$$
\begin{aligned}
& \text { روش شناسى · ل ال درصد. }
\end{aligned}
$$

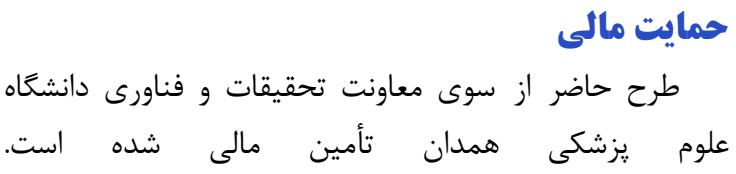

\section{REFERENCES}

1. Jafarian AH, Kooshkiforooshani M, Rasoliostadi A, Roshan NM. Vascular mimicry expression in invasive ductal carcinoma; a new technique for prospect of aggressiveness. Iran J Pathol. 2019;14(3):232. PMID: 31583000 DOI: 10.30699/ijp.2019.94997.1939

2. Tran DP, Spierings EL. Headache and insomnia: their relation reviewed. Cranio. 2013;31(3):165-70. PMID: 23971156 DOI: $10.1179 / \mathrm{crn} .2013 .026$

3. Calder KK, Herbert M, Henderson SO. The mortality of untreated pulmonary embolism in emergency department patients. Ann Emerg Med. 2005;45(3):302-10. PMID: 15726055 DOI: 10.1016/j.annemergmed.2004.10.001

4. Cushman M, Tsai AW, White RH, Heckbert SR, Rosamond WD, Enright P, et al. Deep vein thrombosis and pulmonary embolism in two cohorts: the longitudinal investigation of thromboembolism etiology. Am J Med. 2004;117(1):19-25. PMID: 15210384 DOI: 10.1016/i.amjmed.2004.01.018

5. Roy PM, Meyer G, Vielle B, Le Gall C, Verschuren F, Carpentier F, et al. Appropriateness of diagnostic management and outcomes of suspected pulmonary embolism. Ann Intern Med. 2006;144(3):157-64. PMID: 16461959 DOI: $10.7326 / 0003-4819-144-3-200602070-$ 00003

6. Konstantinides SV, Torbicki A, Agnelli G, Danchin N, Fitzmaurice D, Galie N, et al. 2014 ESC guidelines on the diagnosis and management of acute pulmonary embolism. Eur Heart J. 2014;35(43):3033-69. PMID: 25173341 DOI: 10.1093/eurheartj/ehu283

7. Remy-Jardin M, Pistolesi M, Goodman LR, Gefter WB, Gottschalk A, Mayo JR, et al. Management of suspected acute pulmonary embolism in the era of CT angiography: a statement from the Fleischner Society. Radiology. 2007;245(2):315-29. PMID: 17848685 DOI: 10.1148/radiol. 2452070397

8. Stojanovska J, Carlos RC, Kocher KE, Nagaraju A, Guy K, Kelly AM, et al. CT pulmonary angiography: using decision rules in the emergency department. $J$ Am Coll Radiol. 2015;12(10):1023-9. PMID: 26435116 DOI: 10.1016/j.jacr.2015.06.002

9. Mamlouk MD, vanSonnenberg E, Gosalia R, Drachman D, Gridley D, Zamora JG, et al. Pulmonary embolism at CT angiography: implications for appropriateness, cost, and radiation exposure in 2003 patients. Radiology. 2010;256(2): 625-32. PMID: 20551182 DOI: 10.1148/radiol.10091624

10. Costantino MM, Randall G, Gosselin M, Brandt M, Spinning $\mathrm{K}$, Vegas CD, et al. CT angiography in the evaluation of acute pulmonary embolus. AJR Am J Roentgenol. 2008;191(2):4714. PMID: 18647919 DOI: 10.2214/AJR.07.2552

11. Righini M, Le Gal G, Aujesky D, Roy Pm, Sanchez O, Verschuren F, et al. Diagnosis of pulmonary embolism by multidetector CT alone or combined with venous ultrasonography of the leg: a randomised non-inferiority trial. Lancet. 2008;371(9621):1343-52. PMID: 18424324 DOI: $10.1016 / \mathrm{S} 0140-6736(08) 60594-2$

12. Bettmann MA, Baginski SG, White RD, Woodard PK, Abbara S, Atalay MK, et al. ACR Appropriateness Criteria(R) acute chest pain--suspected pulmonary
ملاحظات اخلاقى

$$
\begin{aligned}
& \text { اين طرح از كميته اخلاق دانشعاه علوم يزشكى همدان به } \\
& \text { شناسه IR.UMSHA.REC.1398.172 تأييديه دارد. }
\end{aligned}
$$

$$
\begin{aligned}
& \text { سهم نو يسندكان } \\
& \text { نويسنده اول (يزوهشخر اصلى): طراحى يروزه، تدوين } \\
& \text { بخشهاى مختلف طرح و ويرايش علمى مقاله . F درصد } \\
& \text { نويسنده دوم (يثروهشخر اصلى): مسئول مكاتبات، تدوين } \\
& \text { يرويوزال، جمع آورى دادهها، بازنگرى متون و نگارش مقاله }
\end{aligned}
$$

embolism. J Thorac Imaging. 2012;27(2):W28-31. DOI: $10.1097 /$

RTI.0b013e31823efeb6

13. Carrier M, Righini M, Djurabi RK, Huisman MV, Perrier A, Wells PS, et al. VIDAS D-dimer in combination with clinical pre-test probability to rule out pulmonary embolism. A systematic review of management outcome studies. Thromb Haemost. 2009;101(5):886-92. PMID: 19404542

14. Pasha SM, Klok FA, Snoep JD, Mos ICM, Goekoop RJ, Rodger MA, et al. Safety of excluding acute pulmonary embolism based on an unlikely clinical probability by the Wells rule and normal D-dimer concentration: a metaanalysis. Thromb Res. 2010;125(4):e123-7. PMID: 19942258 DOI: 10.1016/i.thromres.2009.11.009

15. Stein PD, Woodard PK, Weg JG, Wakefield TW, Tapson VF, Sostman HD, et al. Diagnostic pathways in acute pulmonary embolism: recommendations of the PIOPED II investigators. Am J Med. 2006;119(12):1048-55. PMID: 17145249 DOI: 10.1016/j.amjmed.2006.05.060

16. Chen C, Yu L, Tang X, Liu MZ, Sun LZ, Liu C, et al. Dynamic hip system blade versus cannulated compression screw for the treatment of femoral neck fractures: a retrospective study. Acta Orthop Traumatol Turc. 2017; 51(5):381-7. PMID: 28844681 DOI: 10.1016/j.aott. 2017.07.006

17. Wicki J, Perneger TV, Junod AF, Bounameaux H, Perrier A. Assessing clinical probability of pulmonary embolism in the emergency ward: a simple score. Arch Intern Med. 2001; 161(1):92-7. PMID: 11146703 DOI: 10.1001/archinte.161.1.92

18. Wells PS, Anderson DR, Rodger M, Ginsberg JS, Kearon C, Gent $\mathrm{M}$, et al. Derivation of a simple clinical model to categorize patients probability of pulmonary embolism: increasing the models utility with the Simple RED D-dimer. Thromb Haemost. 2000;83(3):416-20. PMID: 10744147

19. den Exter PL, van der Hulle T, Klok FA, Huisman MV. Advances in the diagnosis and management of acute pulmonary embolism. Thromb Res. 2014;133(Suppl 2):S106. PMID: 24862127 DOI: 10.1016/S0049-3848(14)50002-3

20. Raji H, Javad Moosavi SA, Dastoorpoor M, Mohamadipour Z, Ghanavati SPM. Overuse and underuse of pulmonary CT angiography in patients with suspected pulmonary embolism. Med J Islam Repub Iran. 2018;32:3. PMID: 29977871 DOI: 10.14196/mjiri.32.3

21. Wang J, Wu XY, Liang Y, Guo W. Predictive value of the Wells score combined with D-dimer level in identifying acute pulmonary embolism in patients with coronary heart disease with chest pain. Chinese Med J. 2020;133(18):22535 . 10.1097/CM9.0000000000000988

22. Touhami O, Marzouk SB, Bennasr L, Touaibia M, Souli I, Felfel MA, et al. Are the wells score and the revised geneva score valuable for the diagnosis of pulmonary embolism in pregnancy? Eur J Obstet Gynecol Reprod Biol. 2018;221: 166-71. PMID: 29310042 DOI: 10.1016/j.ejogrb.2017.12.049

23. Vongchaiudomchoke $\mathrm{T}$, Boonyasirinant $\mathrm{T}$. Positive pulmonary computed tomography angiography in patients with suspected acute pulmonary embolism: clinical 
prediction rules, thromboembolic risk factors, and implications for appropriate use. $J$ Med Assoc Thai. 2016;99(1):25-33. PMID: 27455821

24. Molaee S, Ghanaati H, Safavi E, Foroumandi M, Peiman S. Computed tomography pulmonary angiography for evaluation of patients with suspected pulmonary embolism: use or overuse. Iran J Radiol. 2015;12(3):e22383. PMID:
26557282 DOI: 10.5812/iranjradiol.12(2)2015.22383

25. Geeting GK, Beck M, Bruno MA, Mahraj RP, Caputo G, DeFlitch C, et al. Mandatory assignment of modified wells score before CT angiography for pulmonary embolism fails to improve utilization or percentage of positive cases. Am J Roentgenol. 2016;207(2):442-9. PMID: 27144311 DOI: 10.2214/AJR.15.15394. 